\title{
The TrustCoM approach to enforcing agreements between interoperating enterprises
}

\author{
Michael Wilson ${ }^{1}$, Alvaro Arenas ${ }^{1}$, David Chadwick ${ }^{2}$, Theo Dimitrakos ${ }^{3}$, Jurgen \\ Doser $^{4}$, Pablo Giambiagi ${ }^{5}$, David Golby ${ }^{6}$, Christian Geuer-Pollman ${ }^{7}$, Jochen Haller ${ }^{8}$, \\ Stølen Ketil ${ }^{9}$, Tobias Mahler ${ }^{10}$, Lorenzo Martino ${ }^{11}$, Xavier Parent ${ }^{12}$, Santi Ristol ${ }^{13}$, J \\ Sairamesh $^{14}$, Lutz Schubert ${ }^{15}$, Nilufer Tuptuk ${ }^{16}$ \\ ${ }^{1}$ CCLRC Rutherford Appleton Laboratory, \{m.d.wilson, a.arenas \}@rl.ac.uk \\ ${ }^{2}$ University of Kent, d.w.chadwick@KENT.AC.UK \\ ${ }^{3}$ British Telecom, theo.dimitrakos@bt.com \\ ${ }^{4}$ ETH Zurich, doserj@inf.ethz.ch \\ ${ }^{5}$ Swedish Institute of Computer Science, pablo@sics.se \\ ${ }^{6}$ BAe Systems, david.golby@baesystems.com \\ ${ }^{7}$ European Microsoft Innovation Centre, chgeuer@microsoft.com \\ ${ }^{8}$ SAP, jochen.haller@SAP.COM \\ ${ }^{9}$ SINTEF, Ketil.Stolen@sintef.no \\ ${ }^{10}$ NRCCL, tobias.mahler@jus.uio.no \\ ${ }^{11}$ Università degli Studi di Milano, martino@dico.unimi.it \\ ${ }^{12}$ King's College, University of London, xavier@dcs.kcl.ac.uk \\ ${ }^{13}$ Atos Origin, santi.ristol@ATOSORIGIN.COM \\ ${ }^{14}$ IBM, jramesh@US.IBM.COM \\ ${ }^{15}$ High Performance Computing Centre, Stuttgart, Schubert@hlrs.de \\ ${ }^{16}$ Imperial College, University of London, nt102@DOC.IC.AC.UK
}

\begin{abstract}
To respond to market opportunities enterprises must interoperate with each other within dynamic virtual organizations (VO) when they do not control the required resources themselves. The TrustCoM project is developing a framework for trust, security and contract management for dynamic VO. The core contribution of the TrustCoM framework is its ability to define a contractual agreement between VO members at a business level and have it specified, monitored and updated at a technical, operational level within a service oriented architecture. The main innovation in TrustCoM is to apply recent research results on policy based security in distributed computing management, role based access control and reputation management to bridge the gap between VO Agreements and managed Web Services.
\end{abstract}

\section{INTRODUCTION}

In the 1980's the main interoperability challenge for enterprises was to support syntactic interchange of information within organisations. In the 1990's the challenge advanced from syntax and structure to address semantics (e.g., Sheth, 1999). One solution to this is to use service oriented architectures implemented as web services or the grid where service interfaces can be clearly defined. The challenge for the early 
$21^{\text {st }}$ century is an increase in abstraction, to support interoperability within enterprises where non-functional requirements of security and quality of service must be enforced too. Within organizations these non-functional requirements are often stated as service level agreements (SLA), while when the enterprise takes the form of a virtual organization (VO) consisting of multiple legal entities the embodiment of these requirements is as VO agreements, partial contracts or partnership agreements which are supplemented by SLA.

The TrustCoM project ${ }^{1}$ is developing a framework for trust, security, and contract management, for secure, collaborative business processing and resource sharing in dynamically-evolving VO. The term "TrustCoM Framework" stands for the principles and paradigms, the processes and functions, and the architecture and the technology that underpin trustworthy, secure, and contract-driven operations of VO.

The TrustCoM Framework includes the following components:

o a set of semantically well-founded concepts and relationships for describing and reasoning about trust and security in dynamic virtual organisations. This forms the meta-model of the TrustCoM framework;

0 an abstract architecture reflecting these concepts, and providing a flexible structure and organising principles for systems based on the framework;

o profiles extending existing open specifications of services and protocols, or showing how they can be used together to implement the TrustCoM framework.

The TrustCoM framework is heavily based on the proposals of Dimitrakos (2003) for a framework for trust within a service oriented architecture, where the management of risk is the main basis for decisions.

The remainder of the paper describes the vision behind the framework, then some of the key concepts, the architecture, a hint towards the specifications and generic tools to implement it, and then an outline of the practical demonstration and evaluation of the framework. Since the TrustCoM project is only half way through its three years at the time of writing, many issues have been identified but not yet resolved, so the final conclusion outlines some future plans to address these problems.

\subsection{The TrustCoM Vision}

The TrustCoM vision is compatible with others in the world of service oriented architectures, Web Services, the Grid, or the utility/appliance information world. From this general outlook, the TrustCoM vision is distinguished by its focus on trust, contract and security management.

VOs are created when consortia of legal entities wish to work together to produce a product, provide a service or tender for a contract, but do not wish to either have one contracted party to which the others are subcontracted, or to create a new legal entity which they jointly own. VOs can be created quickly, and undertake their role for a

${ }^{1}$ Further details available from http://www.eu-trustcom.com/ 
very brief period of time, or exist for the longer term. VOs can be established by a general VO agreement (GVOA) which outlines the legal framework for the cooperation, within which specific SLA can be produced to detail each service provided by a legal entity within the VO.

When a VO is formed its creator can define a model of the co-operative business it is conducting, and the roles in the business process that need to be cooperatively manned by the potential VO members. Then the VO must discover potential members who can meet these role definitions, negotiate their agreement to the VO agreement, and the individual service dependent SLAs. Within TrustCoM, it is assumed that the descriptions of potential members, and the services to be provided are all available as Web or Grid services, open to automated resource discovery, negotiation and SLA agreement. All potential VO members will register the services that they can offer in a registry, agreeing to the TrustCoM acceptable use policy in order to join an Enterprise Network (EN). The automated resource discovery mechanism operates over the EN registry. Along with the functional definition of the role to be fulfilled, requirements on the quality of service, and cost, one of the factors involved in the selection of VO members will be their previous reputations both to undertake roles defined in the VO business process model, but also to operate under VO agreements and SLAs, and even, their litigiousness.

Once the VO is operating, each participant must open up its internal ICT infrastructure to the other VO members in as much as they require it to undertake their roles in the VO. Consequently there are access control issues for each VO member concerning the authenticated identity of employees of their own and other VO members, as well as authorisation issues of access to data and services throughout each organisation. While a VO is operating, the set of Web or Grid services brought together to achieve its business process model must be monitored and managed to ensure that the cost, time, security and quality measures stated in each SLA are being met. When those terms and conditions stated in the GVOA and SLA are breached, then the reputation of that partner must be updates, and the policies stated in the contract must be enacted to increase the detail of monitoring, restrict access control, fine partners, and even expel and replace non-compliant partners.

When a VO has completed its activity, it must then be terminated to minimise future risks from liability and exposure to security breaches, and ensure the appropriate accounting of expenditure and income and distribution of profit or loss between the participating organisations.

This vision encompasses the collaborative business process model (BPM) of a VO, the roles defined in it, the discovery of organisations to fulfil those roles on the basis of published capability and reputation, the legal agreements between selected organisations to fulfil their roles, the establishment of secure and reliable composition of Web or Grid services to enact the business process models, the monitoring and management of the performance of members, and the decomposition of the VO resulting in minimal outstanding risks. The relation between the executable BPM, SLA management and security concerns all result in policies which must be deployed and monitored during performance to reduce risk within an international legal framework. Therefore each of these components BPM, discovery and negotiation, reputation management, SLA management, security policies, legal framework needs to be analysed, modelled and incorporated into a software system to host dynamic 
virtual organisations where risks and the trust required to offset them can be quantified and judged by business decision makers.

The structure and operation of this infrastructure must also be both transparent, and secure in order to promote trust in the infrastructure itself. To achieve this, in TrustCoM, the framework behind the infrastructure is published, and the source code of a generic implementation of the infrastructure will be published as open source code. A second choice made to increase the trustworthyness of the infrastructure is that the transmission of information within the infrastructure is limited to a minimum - for example, the content of SLA are not promulgated throughout the infrastructure but limited to a discrete SLA management subcomponent.

\section{Conceptual Models supporting the TrustCoM Framework}

The TrustCoM approach to managing $\mathrm{VO}$ is built around the identification of risks to the VO, establishing agreements and SLA that limit those risks, and monitoring the enactment of the VO with respect to terms and conditions in them. This section outlines several difficult conceptual issues associated with the management of VO within the TrustCoM framework. These are all active areas of research to which no clear solution has yet been identified, but where progress is being made.

\subsection{The Virtual Organisation Lifecycle}

TrustCoM follows the life-cycle model developed in the VO roadmap project (Camarinha-Matos and Afsarnabesh, 2003), including the phases of identification, formation, operation/evolution and dissolution. The identification phase covers setting up the VO; this includes selection of potential business partners by using search engines or looking up registries. VO formation deals with partnership formation, including the VO configuration by a VO Initiator, who distributes information such as policies, SLAs, etc, and the binding of the selected candidate partners into the actual VO. After the formation phase, the VO can be considered to be ready to enter the operation phase where the identified and properly configured VO members perform accordingly to their role. Membership and structure of VOs may evolve over time in response to changes of objectives or to adapt to new opportunities in the business environment. Finally, the dissolution phase is initiated when the objectives of the VO has been fulfilled. TrustCoM has added a sub-phase to this last to account for final Termination of the VO in which final liabilities are terminated.

There is also another stage added prior to the VO identification, in which the Enterprise network is established. This provides the set of candidate members for any $\mathrm{VO}$ in a way sometimes referred to as a VO ecosystem or VO nursery.

\section{EN Creation and VO Identification}

The enterprise network creation involves establishing the TrustCoM EN Infrstructure and allowing organisation to register their interest in potential VO.

The identification phase includes defining a Collaborative Business Process Model, where the VO business objective, the business process to achieve this, and the roles required for each service organization are defined, as well as trust, security and contract management (TSC) properties associated to the roles and their interaction. The roles and their TSC properties are used as the base for discovering potential 
business partners from the EN members who are both capable of fulfilling the required roles and of fulfilling the TSC requirements of the VO by using search engines and/or looking up registries.

\section{VO Formation}

During the formation phase the selected set of Members needs to be limited to those who will actually fulfil the roles in the VO, and configured so that they can perform according to their anticipated role in the VO.TSC properties are refined into policies. An important document generated in this phase is the General VO Agreement (GVOA) which record the VO policies as well as the Service Level Agreement (SLA) associated to the services provided by a partners. SLA will be negotiated with each VO member for each service provided.

\section{VO Operation}

This phase can be considered as the main life-cycle phase of a VO. During this phase the identified partners contribute to the actual execution of the VO tasks by executing pre-defined business processes. Important features in this phase are the monitoring of the performance of the $\mathrm{VO}$ as well as the enforcement of policies.

\section{VO Evolution}

VO Evolution is part of the VO Operation phase. When a VO member fails completely or behaves inappropriately, the VO manager may need to dynamically replace such partner. This evolution may involve discovering new business partners, re-negotiating terms and providing configuration information, as done in the identification and formation phases. One of the main problems involved with evolution consists in re-configuring the existing VO structure so as to seamlessly integrate a new partner, possibly even unnoticed by other participants.

\section{VO Dissolution and Termination}

The dissolution phase is carried when the objectives of the VO has been fulfilled. During dissolution, the VO structure is dissolved and final operations are performed to annul all contractual binding of the partners. From a trust and security perspective, this involves resolving federations, revoking security credentials, invalidating VO context information, and updating reputation of all participants.

The final termination of the VO may take place many years after the dissolution since some liabilities may persist after the VO has dissolved. Therefore records must be maintained of the VO membership, in case such liabilities need to be resolved.

\subsection{Trust}

Trust is a difficult concept to achieve a consensual definition of. The English word trust is heavily overloaded with usages, most of which are synonyms to confidence. Such synonyms do not express the unique concept that is itself trust. The computing community has used the term in the last few years in a specialised way to describe authorities whose certificates are accepted as authority of identity or access authority. Within social science, trust has been researched for many years in the context of both personal relationships and economic theory. Trust plays a role in social exchange where it decreases the need for regulation and institutions, and reduces the cost of both transactions within relationships, and the frequency of monitoring the state of a relationship in order to maintain it. Such a role would be beneficial within VO management, but it is important to note that the adoption of trust substitutes such as 
contracts, procedures for monitoring conformance to them, and security mechanisms all reduce the rate of development of trust within relationships.

TrustCoM has adopted the view that trust is a psychological state, comprising the intention to accept vulnerability (often inherent in VO), and based upon positive expectations of the intentions or behaviour of another. Trust exists when one party to a relationship believes the other party has an incentive to act in the interests of the first party, or to give weight to his or her interests when making choices. This is sometimes called the encapsulated interest model of trust relations (Hardin, 2002b), where the importance of interest in maintaining the relationship into the future is the primary foundation of the trustworthiness of each party in the relationship. A trust relation emerges out of mutual interdependence and the knowledge developed over time of reciprocal trustworthiness. From this view of trust, it is argued that distrust can lead to the development of institutions which limit exploitation and protect those without established reputations or trust relationships (Cook, Hardin and Levi, 2005). That is, interpersonal trust is not as imperative in corporate business relationships as has been previously argued in the social science literature.

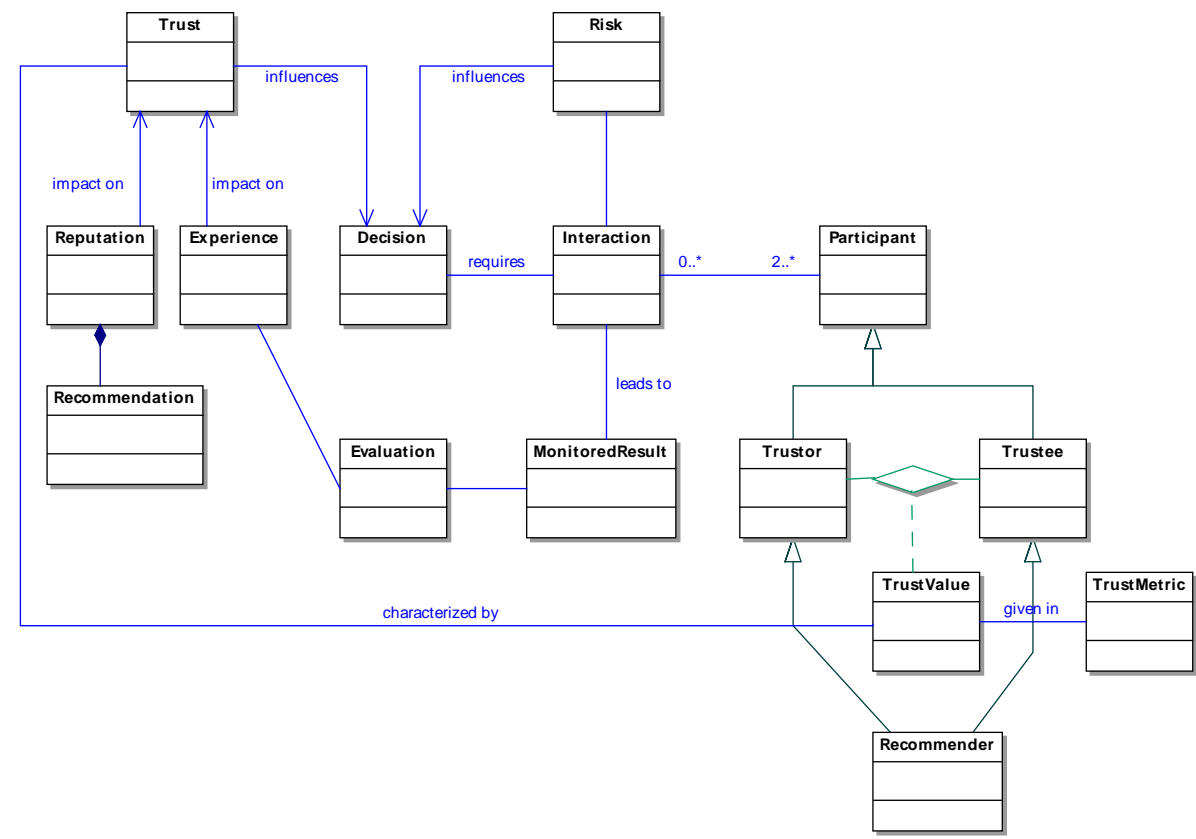

Fig. 1. Conceptual Model for Reputation Management.

The TrustCoM project is still analysing the complex balance between trust and trust substitutes within VO as experience with them grows. Both reduce risks to the VO. We need considerably more data on the details of the interaction of relationships and trust substitutes in virtual organisations before anybody can be confident in the long term consequences of any actions. For example, when short lived VO are formed of individuals, rather than organisations, the $\mathrm{VO}$ provides the only social relationships supporting the VO business. As the size of the enterprises in the VO increase through 
SME to large organisations the relationships between individuals within each partner organisation become more important than personal relationships across VO partners. TrustCoM is considering VO consisting of partners of the SME and larger class where inter-partner relationships are less critical on this scale.

The explicit statement of reputations, business process models, contracts, SLA's and other details of the business process should all act to support mutual knowledge between contracting parties and hence act as institutions to provide a basis for trust.

One consequence of this view of trust as a goal based concept is that it can be used to resolve issues of policy conflict resolution where goal based conflict resolution is one option, contrasted with utility based resolution. It should also be noted that this analysis considers trust across the VO itself, rather than detailed issues of user interface design to promote trust between individual users. This topic is an active area of research outside the project (e.g. Riegelsberger, Sasse and McCarthy, 2005) whose results will be incorporated into detailed user interface design choices.

\subsection{Collaborative Business Process Model}

The collaborative Business Process Model (BPM) defines the business objective and the process to achieve it. Since the model applies across multiple organisations it is an extension of existing BPM technologies. It defines each service and the role of an organisation that would provide that service. The role definitions can then be used as input into the discovery service to identify possible VO members.

\subsection{Reputation Management}

Members in the VO will need to carry out reputation management, e.g. qualifying their trust relationships with other entities in the VO. This information can be used to learn more about the behaviours of the entities in the $\mathrm{VO}$ and to make trust decisions about existing and new entities in the VO.

Figure 1 shows the core conceptual model of reputation management. In this view, whenever a step in the BPM is completed, the resulting achievements are recorded for each role that an agent has fulfilled in terms of SLA conditions - that is, was the work done on time, to budget and to quality. This data provides a basis for judging whether an organization has been capable of fulfilling a role in the past. The judgment then required is whether past performance is an indicator of future performance. As many financial services advertisements are legally obliged to state - this is not always the case. Consequently, different models are available to relate past performance in one role to future performance in that, or other related roles - consistency and generalization being the crucial concepts. The reputation management systems supports alternative algorithms that can be selected to make decisions on the stored data.

A further problem being pursued is the management of the reputation of reputation management services themselves - is it inevitable that such services become conservative in their judgements in order to maintain their own reputations, at the cost of innovation? 


\subsection{Service Discovery and VO partner business negotiation}

UDDI provides a very simple registry for Web Services that can be searched by their functional properties. One much vaunted approach to enrich such discovery has been through Semantic Web descriptions of the qualities of the services using OWL-S or WSMO as the basis for description. Although these leave room for quality of service descriptions, neither address the range of descriptions that TrustCoM would require to address competence, reputation, cost and other negotiable SLA measures to describe and select the best organisation to fulfil a role in a VO. Consequently, TrustCoM is extending the UDDI registry description to include these aspects independently of the Semantic Web approach in the expectation that the two approaches will later merge once the conceptual issues are resolved.

The algorithms for service discovery are also unclear - since different strategies for forming a VO can be chosen to manage different risks. For example, the risk that the termination of one organisation may have too large an impact on the VO would result in limiting the number of roles that any single organisation could play; alternatively, the risk that an organisation could hold the VO to ransom for its role may require a partner selection strategy that the skills to support each role should be provided by at least two organisations. Given these alternative strategies, the same approach is taken as with reputation management, that alternative strategies for negotiating partner selection can be implemented within a generic engine.

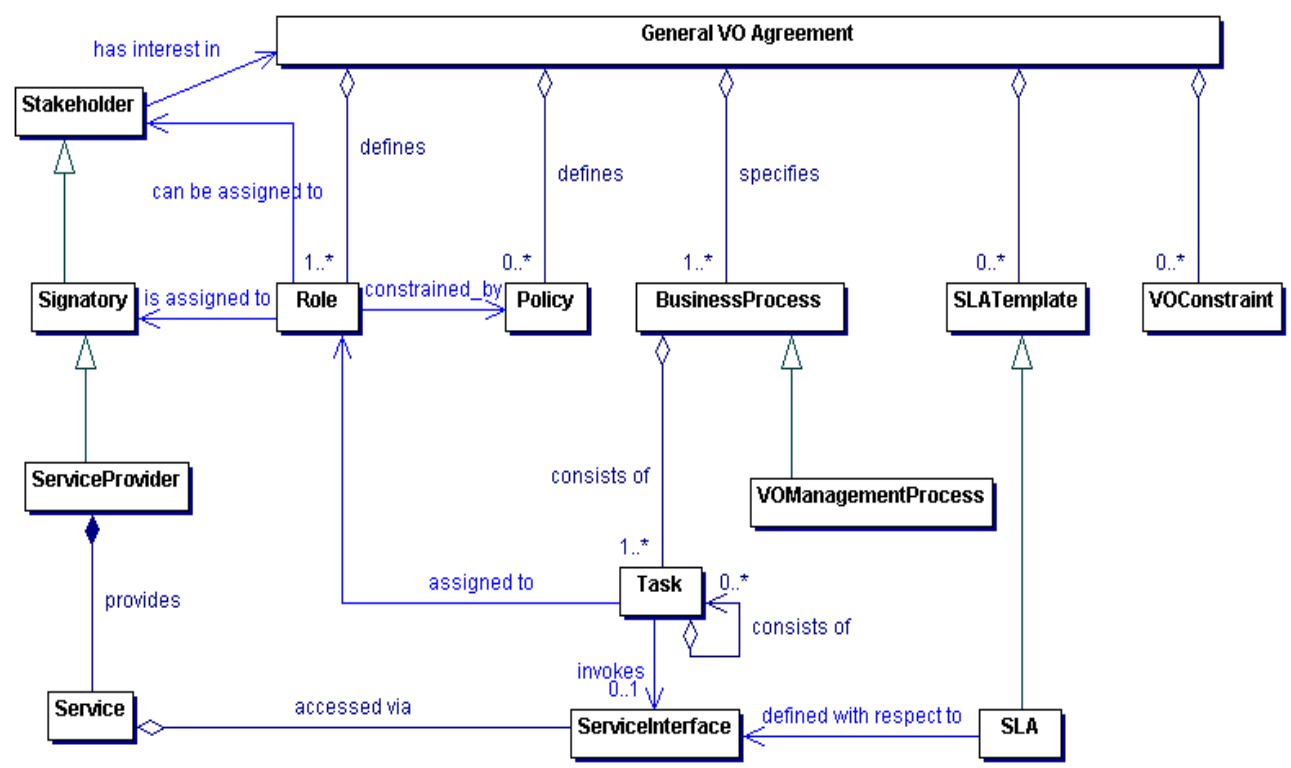

Fig. 2. Static Model of the General VO Agreement.

\subsection{The General VO Agreement}

The general VO agreement is signed by all VO members when the VO is created. The agreement is created from template section based on Weitzenboeck (2001) structured as shown in figure 2. 
The VO agreement includes the legal aspects of the VO relationship which are introduced to account for legally identifiable risks, so TrustCoM includes the development of a legal risk analysis tool to provide an accessible mechanism to choose the appropriate clauses. Constraint clauses are described in both natural language and a machine readable XML format so that they can be loaded into the TrustCoM tools as policies.

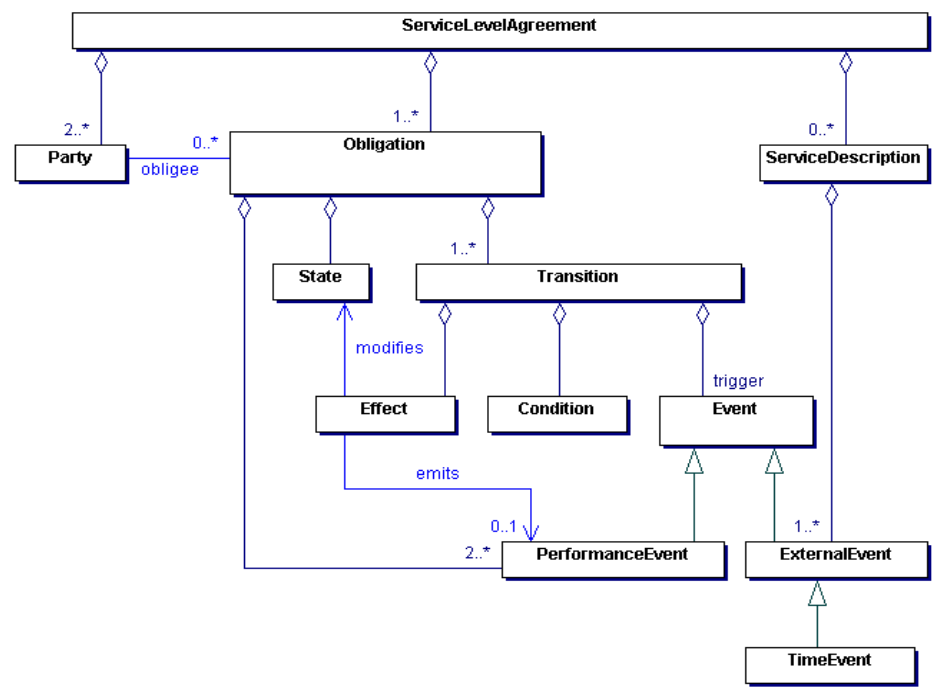

Fig. 3. Static Model of a Service Level Agreement.

\subsection{VO Service Level Agreements}

For each service in the VO, an SLA is required to define the performance indicators of the service. Like the GVOA, the structure of the SLA is represented in XML combining text and machine readable policies that can be loaded into tools. Figure 3 presents a static model of an SLA.

\section{The TrustCoM Architecture}

The TrustCoM Framework is implemented within a general service oriented architecture supporting Web and Grid Services. The layered structure of the main components on top of this Web Services (WS) foundation is shown in Figure 4. The main block of components between WS Foundation and Federation are the generic TrustCoM components for managing Web Services. On top of this are the two final layers which are specific to VO management and the specific application domain of a particular VO. The VO management layer has much in common with the main control loop of the application, cycling through the VO lifecycle. Figure 5 shows how the VO management component sits in the midst of the interactions of the major components. 


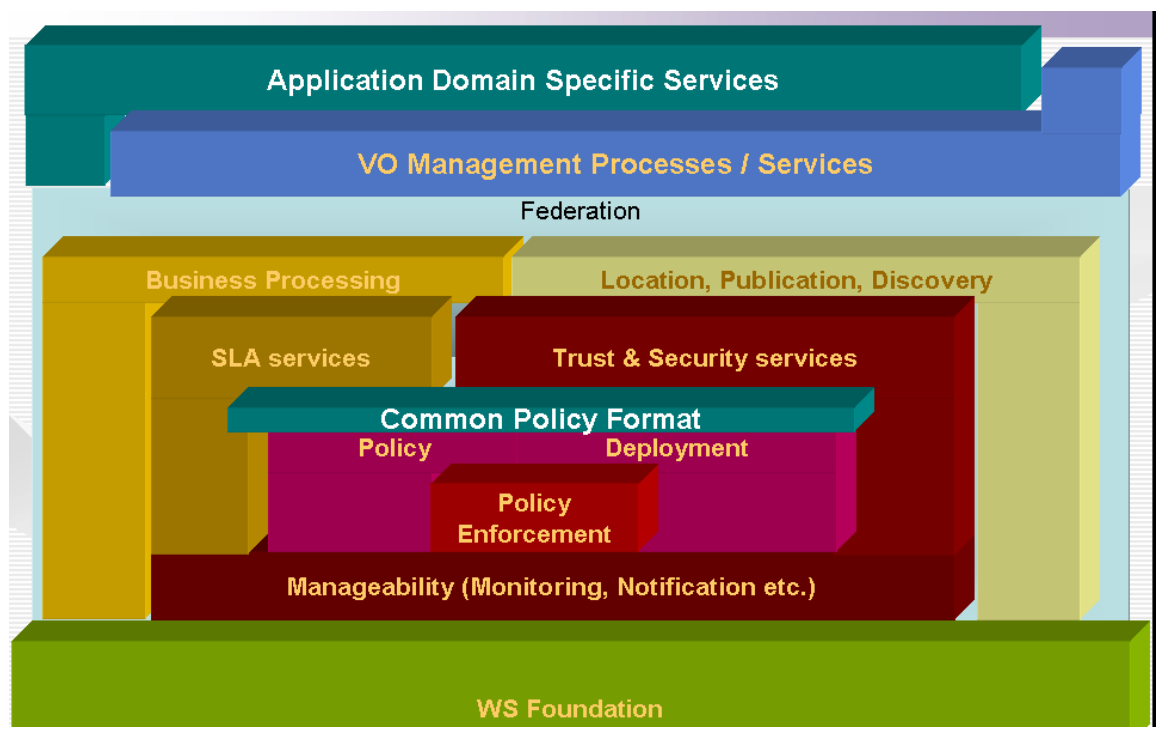

Fig. 4. The major components of the TrustCoM architecture.

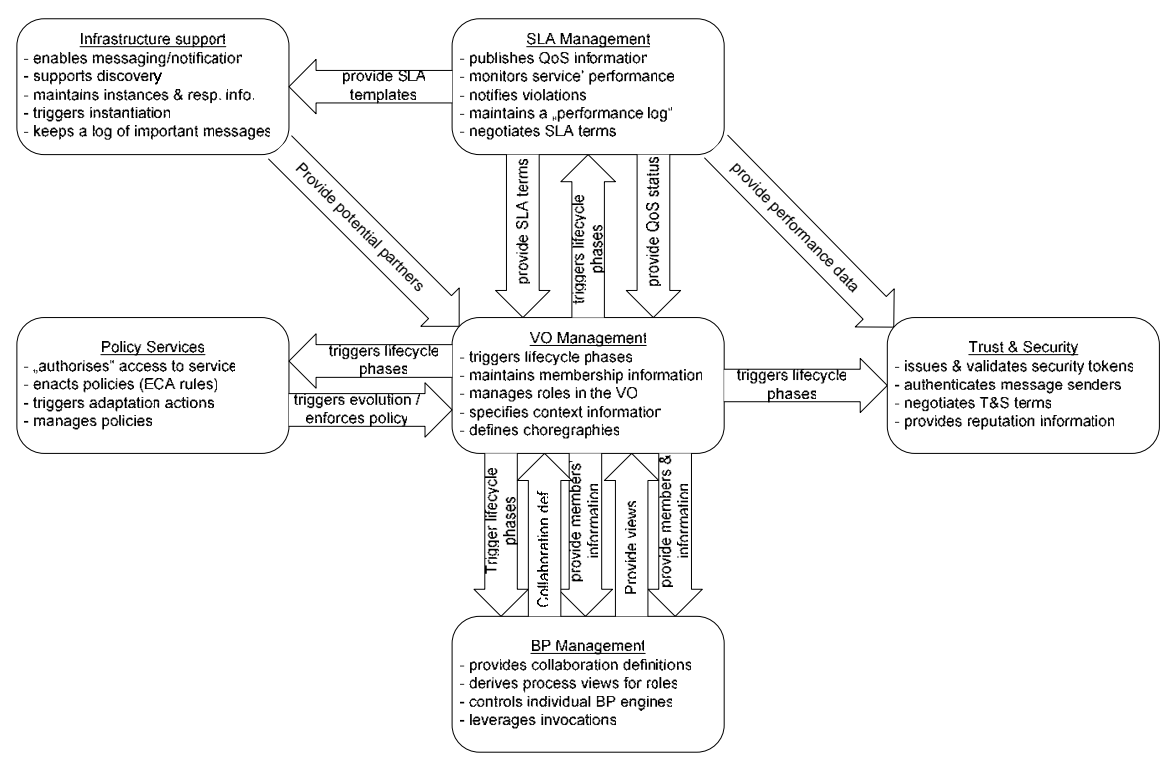

Fig. 5. The interactions of the major architecture components. 


\section{Demonstrating and Evaluating the TrustCoM Framework}

Two scenarios from the many considered by TrustCoM are being used as concrete demonstrations of the TrustCoM Framework, and the application of the generic tools. These arise from different and complimentary requirements which provide a broad basis for evaluating the TrustCoM framework and tools. One is in the area of distributed aerospace engineering, while the other is in the area of an e-learning application provided by a distributed service provider.

\subsection{Distributed aerospace engineering application}

The aerospace industry has a history of collaborative projects usually managed through sub-contract relations from a large coordinator company. However, products are becoming increasingly more difficult to produce and support due to their complexity. Consequently joint ventures are an increasingly adopted approach to reducing risk and allowing companies to concentrate on their core competencies. In order to maximize the benefits of the flexibility available in VO's these relationships are become more transitory in order to respond to the rapidly changing market needs, resulting in a greater emphasis on the integration role. Issues that arise include: controlled sharing of resources, co-ordination of processes, controlled visibility of processes, interdependency of processes, sharing of decisions, sharing of responsibilities and liabilities, and the use of agreements in order to limit those liabilities.

\subsection{An e-learning marketplace application}

Existing telecom service providers believe that they can provide added value services to companies by providing a forum for them to form VO to undertake large contracts through automating the product value chain. This is a new business opportunity for the service provider which automates and globalizes relationship building. As we move more into a knowledge economy the class of business to be supported includes more purely knowledge based businesses such as e-learning. In this scenario, a marketplace is provided for e-learning courseware and materials developers to join together to provide courses to meet the needs of individuals and organizations who can analyse their existing skills, and compare them with the skills required for potential business opportunities, identifying the gap to be filled through the e-learning activity.

The contrast between the existing and new relationship management, the long and short term duration of the enterprise, the size of the member organizations etc.. between these two applications should allow them to provide a broad range of test problems to evaluate the technology, business models, contract construction and enforcement aspects of the TrustCoM Framework. Both applications are currently under development and results are not expected for several months. 


\section{Conclusion and Future Work}

The TrustCoM project is half way through its three year duration, in which time it has specified the concepts that it is addressing, the architecture to implement it, the components of that architecture and applications to

The project is currently developing the software for the generic tools and applications, which can then be evaluated to feed back into the framework design and presentation. In parallel conceptual work is still going on to address those research issues, business models, and legal issues where commitments have not been made.

\subsection{Acknowledgments}

The work reported in this paper has been partially funded by the EC through an IST programme grant to the TrustCoM integrated project.

\section{REFERENCES}

1. Camarinha-Matos, L.M. and Afsarmanesh, H. (2003) A Roadmap for Strategic Research on Virtual Organisations, in Proceedings of PRO-VE 2003, 33-46, Kluwer.

2. Cook, K.S., Hardin, R., Levi, M. (2005) Cooperation without trust ? New York: Russell Sage Foundation.

3. Dimitrakos, T. (2003), A Service-Oriented Trust Management Framework , in Proc. International Workshop on Trust in Agent Societies, Melbourne, Australia, 14-15 Jul 2003, Trust, Reputation and Security: Theories and Practice

4. Dimitrakos, T., Golby, D. and Kearney, P. (2004) Towards a trust and contract management framework for dynamic virtual organizations. In eAdoption and the Knowledge Economy: Proceedings of e-challenges 2004.

5. Hardin, R (2002) Trust and Trustworthiness. New York: Russell Sage Foundation.

6. Riegelsberger, J., Sasse M.A., and McCarthy, J.D, (2005) The mechanics of trust: A framework for research and design. Int J. Human-Computer Studies, 62, 381-422.

7. A. P. Sheth, (1999) Changing focus on interoperability in information systems: from system, syntax, structure to semantics. In M.F. Goodchild, M.J. Egenhofer, R. Fegeas and C.A. Kottman (eds) Interoperating Geographics Information Systems. Kluwer.

8. Weitzenboeck, E. (2001) Electronic Agents and the Formation of Contracts, International Journal of Law and Information Technology, 9 (3) Oxford University Press, pp. 204-234. 